\section{Über den Tellerrand}

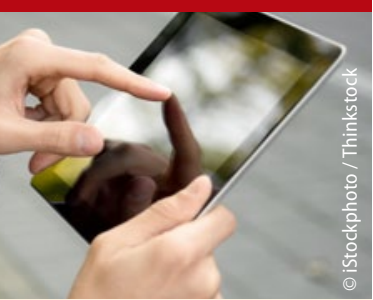

- European Spine Journal

Top Downloads

Das internationale Springer Journal European Spine Journal bietet einen umfassenden Überblick über Forschung, Diagnose und Behandlung der Wirbelsäule. Diese Beiträge wurden im Juli online am häufigsten gelesen:

- A systematic review with metaanalysis of posterior interbody fusion versus posterolateral fusion in lumbar spondylolisthesis - Antibiotics a cure for back pain, a false dawn or a new era?

- Antibiotic treatment in patients with chronic low back pain and vertebral bone edema (Modic type 1 changes): a double-blind randomized clinical controlled trial of efficacy

Die Zeitschrift ist offizielles Organ von EuroSpine, der europäischen Wirbelsäulengesellschaft, und The Bone and Joint Decade 2010-2020, sowie weiterer internationaler Fachgesellschaften.

Der Impact Factor 2012 ist 2,133.

Sie finden diese Beiträge über die

Suchfunktion auf

www.springermedizin.de

Als Abonnent von Springer Medizin e.Med können Sie alle Beiträge online im Volltext lesen.

- Springer Medizin e.Med bietet Ihnen:

- Online-Zugriff auf über 600 Fachzeitschriften

- 500 CME-Beiträge für Ihre Fortbildung

- auf Wunsch eine gedruckte Fachzeitschrift

— „Ärzte Zeitung digital“ für iPad

Weitere Infos und das kostenlose Testangebot finden Sie unter www.springermedizin.de/eMed

Orthopäde 2013 · 42:988

DOI 10.1007/s00132-013-2205-y

(c) Springer-Verlag

Berlin Heidelberg 2013

\title{
Entwarnung
}

\section{Kaffee schlägt nicht auf die Knochen}

Ein lange gehegter Verdacht im Zusammenhang mit übermäßigem Kaffeekonsum konnte jetzt widerlegt werden: Das Risiko für osteoporotische Frakturen scheint der Bohnensaft, auch in rauen Mengen genossen, nicht zu erhöhen.

Schwedische Forscher haben in einer großen Longitudinalstudie mit 61.433 Frauen der Geburtsjahrgänge 1914 bis 1948 untersucht. Die Daten stammen aus der Swedish Mammography Cohort. Bei Studienbeginn im Jahr 1987 waren die Frauen im Mittel zwischen 50 und 57 Jahre alt.

Innerhalb einer Beobachtungszeit von durchschnittlich 14 Jahren hatten 14.738 Frauen Frakturen erlitten, davon 3871 an der Hüfte. Die Multivariablen-Analyse ergab jedoch keinen Zusammenhang zum Kaffee- konsum: Kaffeetrinkerinnen brachen sich im Studienzeitraum nicht signifikant häufiger die Knochen als Verächterinnen des Genussmittels ( $<1$ Tasse täglich). Die Hazard Ratio (HR) für Frakturen insgesamt lag bei 0,99, die für Hüftfrakturen bei 0,97 , bezogen jeweils auf eine Tasse (ca. $200 \mathrm{ml}$ ) Kaffee täglich.

$A b$ vier Tassen Kaffee sank die Knochendichte in der Lendenwirbelsäule um $4 \%$, im proximalen Oberschenkelknochen um 2\%. Das relative Osteoporoserisiko gegenüber Nichtkonsumentinnen lag bei den Vieltrinkerinnen bei 1,28 , und zwar nachdem man eine ganze Latte möglicher Einflüsse herausgerechnet hatte (BMI, Aufnahme von Kalzium, Vitamin D, Kalium, Phosphor und Protein mit der Ernährung, Alkohol, körperliche Aktivität, Komorbiditäten, Kortisoneinnahme, Hormon- ersatztherapie, Therapie mit Bisphosphonaten). Die Odds Ratio pro 200-ml-Tasse betrug 1,06.

Ausgeprägter Kaffeekonsum mag $\mathrm{zu}$ einer geringfügigen Abnahme der Knochendichte führen. Dies schlägt sich jedoch offenbar weder in einem nennenswert erhöhten Osteoporoserisiko noch in puncto Frakturen nieder, so die Wissenschaftler. Damit könne man die über Jahre geführte Debatte um Kaffee als möglichen Risikofaktor für osteoporotische Frakturen als beendet betrachten, schreiben Hallström und Kollegen. (eo) Quelle: www.springermedizin.de

In unseren Dossiers „Ernährung“ und „Osteoporose“ finden Sie weitere Beiträge

- www.springermedizin.de/ api-ernaehrung

- www.springermedizin.de/ orthopaedie-osteoporose

\section{Online miträtseln: Blickdiagnose interaktiv Was verursacht diese „Rattenbisse“?}

Bei diesem Mann haben sich diese Veränderungen an einzelnen Fingern gebildet.

Was ist die Ursache dieser rattenbissartigen Ulzerationen?

- Systemische Sklerodermie

- Nephrogene systemische Fibrose

- Skleroderma diabeticorum

- Erfrierung

- Porphyrie

Lösung

Gehen Sie auf www.springermedizin.de/4411086 und klicken

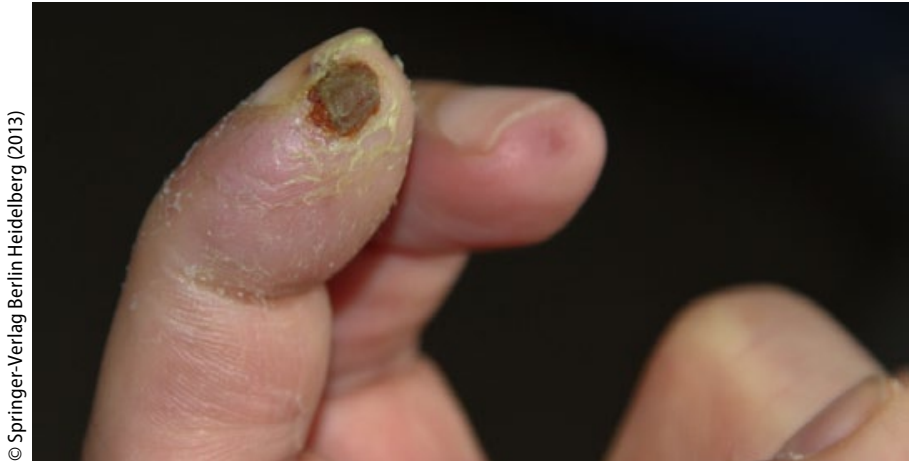

\ Digitale Ulzeration, sog. Rattenbissnekrosen

Sie auf dem Beitrag Ihre Lösung an. Dort erfahren Sie sofort, ob Sie richtig liegen - nebst interessanten Infos zu diesem Fall.
Rätseln Sie

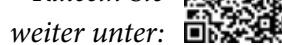

- www.springermedizin.de/ blickdiagnose-interaktiv 